\title{
Anesthesia considerations for robotic-assisted laparoscopic prostatectomy: a review of 1,500 cases
}

\author{
Michael J. Danic · Matthew Chow - Gaylord Alexander • \\ Akshay Bhandari · Mani Menon · Morris Brown
}

Received: 15 February 2007 / Accepted: 29 April 2007/Published online: 30 May 2007

(C) Springer London 2007

\begin{abstract}
Prostate cancer is the second most common cancer in American men and new surgical techniques have led to less invasive options for prostate surgery. Innovations in robotic technology have enabled robotic systems to become a more common sight in operating theatres throughout the United States. Approximately 1,500 consecutive patients scheduled for elective robotic prostatectomy (RP) were reviewed. Patient demographics were recorded and significant intraoperative and postoperative events were reviewed. At our institution the mean age for patients undergoing RP is 60.3 (41-79) years; the mean body mass index is $27.3 \mathrm{~kg} \mathrm{~m}^{-2}$. The mean operative time, defined as the time from the start of insufflation to closure is 177.5 (81-365) $\mathrm{min}$ and mean blood loss is 109 (50750) $\mathrm{mL}$. There was a $1.3 \%$ incidence of postoperative anemia (hemoglobin $<10 \mathrm{~g} \mathrm{dL}$ ) where patients required blood transfusions $(15 / 1,500)$. Three patients were diagnosed with postoperative pulmonary emboli and were
\end{abstract}

This review has not been submitted to any other journal or presented at any conference. All the authors have read and contributed to the manuscript. The authors give full consent for submission and publication. There are no sponsors or financial obligations to report.

M. J. Danic $(\varangle) \cdot$ M. Chow · G. Alexander .

M. Brown

Department of Anesthesiology, Henry Ford Hospital,

2799 West Grand Blvd, Detroit, MI 48202-2689, USA

e-mail: mdanic1@hfhs.org

A. Bhandari

Vattikuti Urology Institute, Henry Ford Hospital,

2799 West Grand Blvd, Detroit, MI 48202-2689, USA

M. Menon

Department of Urology, Vattikuti Urology Institute, Henry Ford

Hospital, 2799 West Grand Blvd,

Detroit, MI 48202-2689, USA treated with IV heparin with no additional sequelae. The most common anesthesia-related complication was corneal abrasions, which were seen in $3 \%$ of cases. One patient required postoperative mechanical ventilation because of laryngeal edema secondary to multiple intubation attempts from an unexpectedly difficult airway. Anesthetic and perioperative complications are rare for patients undergoing robotic-assisted laparoscopic prostatectomy at our institution. Our institution has performed more robotic prostatectomies than any other institution in the world and we review our experience delivering anesthesia for the first 1,500 patients undergoing this operation.

Keywords Prostatectomy - Steep Trendelenburg · Vattikuti

\section{Introduction}

With the exception of non-melanoma skin cancer, cancer of the prostate is the most common cancer in American men. In 2006, it was estimated there were over 234,460 new cases of prostate cancer diagnosed, with over 27,350 deaths attributable to the disease, in the United States [1]. Since the widespread use of the prostate specific antigen (PSA) test, prostate cancer has been detected earlier, usually presenting as elevated serum PSA [2].

Options for the patient with early prostate cancer include surgery, radiation therapy, and watchful waiting [3]. Radical prostatectomy is the only treatment shown in randomized clinical trials to improve disease-specific survival and overall survival, however [4]. For patients who undergo traditional radical prostatectomy, complications include urinary incontinence and impotence, both because of damage to the urinary sphincter and penile nerves [3]. 
These adverse effects can cause significant changes in quality of life and discourage patients from seeking surgical intervention.

The advent of minimally invasive surgical treatment of prostate cancer has increased patient acceptance of this option, despite the absence of randomized clinical trials showing significant advantages of this approach [5]. Technological improvements have recently enabled robotic manipulation techniques to become a more common sight in operating theatres throughout the United States. Robotic prostatectomy (RP) utilizing the da Vinci surgical system (Intuitive Surgical, Sunnyvale, CA, USA) has been performed at our institution since October 2000. The da Vinci system is an elegant and sophisticated "master-slave" robot incorporating advanced three-dimensional optics with three robotic arms. One of the arms controls an endoscope and the other two arms are "slaved" to two control handles housed in mobile control console [5]. The Vattikuti Institute prostatectomy or VIP is a method of RP developed by surgeons at Henry Ford Hospital that involves extensive nerve sparing techniques utilizing both transperitoneal and extraperitoneal dissection. Since the first case in 2001, more than 40,000 operations have been performed worldwide. Our institution has performed more RPs than any other institution in the world and in 2006 more than 30,000 patients underwent the VIP RP worldwide. With Institutional Review Board Approval and waived consent, we review our experience delivering anesthesia for the first 1,500 patients undergoing this operation.

\section{Preoperative evaluation}

Patients undergo a complete history and physical with particular attention to cardiovascular, pulmonary, and endocrine co-morbidities that affect men who are at highest risk of prostate cancer (40-80 years old). At our institution the mean age for patients undergoing RP is 60.3 (41-79) years. Routine preoperative evaluation includes electrocardiography, chest X-ray, hematocrit, and electrolyte screening which is used to aid identification of underlying myocardial ischemia, chronic obstructive pulmonary disease, anemia, and hyperglycemia. This patient population has an increased likelihood of tobacco abuse, coronary artery disease, and renal abnormalities secondary to prostatic obstruction. Low functional status or any signs suggestive of cardiac failure may lead to further cardiovascular testing including transthoracic echocardiogram for evaluation of systolic or diastolic dysfunction, segmental wall motion abnormalities, or valvular abnormality. Underlying pulmonary dysfunction, particularly chronic obstructive pulmonary disorder, places the patient at higher risk for this laparoscopic procedure, that often results in high peak airway pressures to overcome the large intra-abdominal pressure (IAP) increases seen with pneumoperitoneum $(\mathrm{PnP})$ and steep Trendelenburg position [6]. At our institution any patient who is a suitable candidate for conventional retropubic (open) radical prostatectomy is a candidate for RP.

Obese patients (body mass index $>30 \mathrm{~kg} \mathrm{~m}^{-2}$ ) require special consideration, because these patients may have an unexpectedly difficult airway, and are at higher risk of coronary artery disease, pulmonary dysfunction, and diabetes [7-9]. Mean body mass index in these 1,500 patients was $27.3 \mathrm{~kg} \mathrm{~m}^{-2}$. Because of technical difficulties associated with increased body weight, in our earlier experience we observed an increase in surgical time. With our current experience, however, operative times are not significantly different, nor does there seem to be any increased risk of either blood transfusion or prolonged hospital stay.

\section{Preoperative medications}

All patients are instructed to be on a clear liquid diet starting one day before surgery and to stop taking anything orally after midnight preceding the surgery [6]. Although patients may take home medications, diabetic patients are typically instructed to withhold morning insulin doses and oral hypoglycemics secondary to their nil per oral (NPO) status. For diabetic patients, strict glycemic control (80$110 \mathrm{mg} \mathrm{dL}^{-1}$ ) is maintained in the perioperative period. Aspirin and antiplatelet medication are held for two weeks before the procedure, because even the slightest bleeding may obscure the surgical field [6]. A full bowel preparation is not necessary, but patients are required to use a laxative on the day before surgery [6].

Patients with gastroesophageal reflux are pretreated with an H2-antagonist (ranitodine/famotidine) and/or a nonparticulate antacid (sodium citrate), preoperatively, to reduce the risk of complications associated with aspiration pneumonitis. Preoperative antibiotic prophylaxis is given within $1 \mathrm{~h}$ of incision. We use $1-2 \mathrm{~g}$ cefazolin or $1 \mathrm{~g}$ vancomycin for penicillin-allergic patients. Preoperative administration of 5,000 $\mathrm{U}$ subcutaneous heparin and sequential compression devices (SCDs) are used for deep venous thrombosis prophylaxis.

\section{Monitoring}

Because of relatively short operative times (insertion of pneumoinsufflation needle to closure of incision) of 177.5 (81-365) min and minimal blood loss (mean blood loss is $109(50-750) \mathrm{mL}$ at our institution), invasive monitoring for VIP patients is rarely indicated. We have found that one freely flowing peripheral IV and plethysmography offer necessary access and hemodynamic information. If a perioperative cardiovascular emergency arises, trans- 
esophageal echocardiography is the most effective means of assessing cardiac function. With regard to these recommendations, it may be prudent at institutions becoming familiar with RP, and where prolonged operative times are expected, to place invasive monitors preoperatively in higher risk patients, because access to the patient is limited as soon as the robot is docked.

\section{Intraoperative considerations}

Induction and maintenance anesthesia

Any induction agent may be used if it is consistent with the patient's cardiovascular history and other co-morbid conditions. Although choice of inhalation agent or non-depolarizing muscle relaxant is unimportant, complete muscle relaxation is essential. This facilitates ease of mechanical ventilation, introduction of surgical equipment, and creation of PnP that enables optimal surgical working space and visualization. An early indication of inadequate muscle relaxation may be collapse of the PnP. Preoperative epidural insertion for postoperative pain management is not recommended, because this procedure is minimally invasive.

Positioning

A standard electrically operated or motorized table is used to facilitate maneuvering of the patient into surgically favorable positions. Cushioned stirrups are used to place the patient in a modified lithotomy position. Arms and hands are generously padded before they are tucked at the patient's sides. IV access and blood pressure cuff should be rechecked and working in optimal fashion after the arms are tucked, because of limited access after completion of patient positioning. RP surgical patients are prone to slipping off the operating table during table position changes, particularly in the $45^{\circ}$ steep Trendelenburg position. The patient is, therefore, well strapped to the operating room table with chest binding in an " $x$ " like pattern. Particular attention should be paid to pressure areas, because of limited access and the extended duration of the procedure. Both ulnar neuropathy and lateral femoral cutaneous nerve injury may occur if the arms or legs are improperly positioned and/or inadequately padded. Preoperative evaluation should focus on any pre-existing nerve injury with detailed documentation. Any suspected perioperative nerve injury should be investigated immediately with electromyography and nerve conduction studies to provide valuable clinical information.

\section{Laparoscopy}

At our institution all VIP procedures utilize carbon dioxide $\left(\mathrm{CO}_{2}\right)$ for PnP insufflation. Initially the abdomen is inflated to achieve an IAP of $20 \mathrm{~mm} \mathrm{Hg}$ to facilitate safe port placement. When the laparoscopic ports have been placed, IAP may be decreased to $12-15 \mathrm{~mm} \mathrm{Hg}$ for the remainder of the procedure.

Pneumoperitoneum is associated with multiple cardiovascular effects during laparoscopy and these changes may be exacerbated in RP patients in the steep Trendelenburg position. Previous studies have shown that an increase in IAP results in an increase in systemic vascular resistance, mean arterial pressure (MAP), and heart rate (HR), while stroke volume decreases [10]. We have, however, found that combination of the $45^{\circ}$ steep Trendelenburg position and insufflation pressures of $20 \mathrm{~mm} \mathrm{Hg}$ result in a MAP reduced by $17 \%$, HR reduced by $21 \%$, and cardiac output reduced by 37\% (Chow M et al., March 2004, Michigan Society of Anesthesiologists). These cardiovascular changes may have adverse consequences in patients with limited cardiac reserve and invasive monitoring may be necessary if prolonged operative times are expected. PnP also results in impairment of respiratory mechanics [10]. Cephalad migration of the diaphragm and an increased IAP cause peak and plateau pressures to rise by more than 50\% [10]. Pulmonary compliance is reduced and this continues even after desufflation. We have found that chest binding, steep $45^{\circ}$ Trendelenburg position, and high insufflation pressures reduces pulmonary compliance by $68 \%$. These pulmonary changes can have significant adverse consequences for patients with abnormal pulmonary mechanics. Absorption of carbon dioxide during prolonged $\mathrm{PnP}$ may also lead to hybercarbia, which may be treated with increases in ventilation rate. Although no patients required conversion from VIP to open prostatectomy for high peak airway pressures, $0.1 \%$ of cases $(2 / 1,500)$ have been converted because of excessive intra-abdominal adhesions which obscured proper visualization.

Preoperative and intraoperative intravenous fluids are kept to a minimum $(<2,000 \mathrm{~mL})$, because excessive urine output can obscure the operative field during bladder neck transection and urethrovesical anastomosis. Fluid restriction also minimizes facial edema that can result from prolonged use of the steep Trendelenburg position. Pharyngeal and laryngeal edema may also be associated with prolonged steep Trendelenburg position and an endotracheal tube cuff leak test may be warranted before extubation. Because patients are volume-depleted by the conclusion of the procedure, it is important to restore hydration as soon as possible. We have identified that when the urethrovesical anastomosis is complete and the patient is returned to the supine position, intravenous fluids can be restored rapidly with a 1-L bolus of lactated Ringer's and then infusion at $150 \mathrm{~mL} \mathrm{~h}^{-1}$ for the next $12-24 \mathrm{~h}$, depending on the patient's volume status. Intravenous ketorolac $15-30 \mathrm{mg}$ is administered to all patients with 
normal renal function approximately $30 \mathrm{~min}$ before reversal of anesthesia for postoperative analgesia.

\section{Postoperative considerations}

We analyzed the results of the first 1,500 VIPs performed by three different surgeons at our institution. Most patients undergoing VIP are discharged home within $24 \mathrm{~h}$ of surgery (92\%). Mean hospital stay is 1.2 days [11]. To enhance discharge readiness and to maintain functionality patients are encouraged to ambulate within a few hours of surgery. All of the benefits of minimally invasive surgery apply to VIP. These include less postoperative pain, cosmetic incisions, and shorter hospitalization. Intravenous ketorolac and acetaminophen with codeine are usually sufficient for pain control. Immediate postoperative surgical pain should be distinguished from bladder spasms, which are managed with an opium and belladonna rectal suppository. Patients are started on a clear liquid diet immediately postoperatively and diet is advanced as bowel function returns. Urine output is a useful surrogate for assessing hydration status and fluid boluses (500 mL lactated Ringers) are given if urine output decreases to $<0.5 \mathrm{mg} \mathrm{mL} \mathrm{h}{ }^{-1}$. SCDs, subcutaneous heparin, and early ambulation all help to reduce the incidence of deep-vein thrombosis (DVT) and subsequent pulmonary embolism (PE).

\section{Postoperative complications}

Postoperative ileus, abdominal distention, or nausea were the most common complications, with an incidence of $1.7 \%$. Most patients can be managed conservatively, with intravenous fluids and bowel rest, whereas some patients may require gastric decompression with a nasogastric tube. Approximately $0.6 \%(10 / 1,500)$ patients required additional surgical intervention during their hospital stay. Three patients required treatment for bowel injury, three for port site hernias, three for intrabdominal bleeding, and one for anastomosis revision. There was a $1.0 \%(15 / 1,500)$ incidence of postoperative anemia (hemoglobin $<10 \mathrm{~g} \mathrm{dL}^{-1}$ ) in which patients required blood transfusion. Approximately $0.2 \%(3 / 1,500)$ patients were diagnosed with postoperative pulmonary emboli and were treated with IV heparin with no additional sequelae.

The most common anesthesia-related complication was corneal abrasion, which was seen in $3 \%$ of cases, despite the use of eye tape. We were concerned that the extensive degree of the Trendelenburg position put the patient at risk of coming into contact with monitoring cables and we now use eye patches for all patients. With the use of eye patches we have been able to reduce the incidence of corneal abrasions to $1 \%$. Symptoms from corneal abrasions were transient and there were no adverse long-term sequelae. One patient developed forearm swelling secondary to intravenous infiltration. The procedure was aborted, because of concern about compartment syndrome, and was completed the next day. One patient who had a very difficult intubation developed postoperative bronchial edema and required continued intubation overnight without further sequelae.

Robotic failure has occurred perioperatively in $0.2 \%$ $(4 / 1,500)$ of cases. In one case the da Vinci surgical system failed intraoperatively and a substitute robot was used. Three other cases were delayed intraoperatively secondary to software failures, all of which were resolved in the operative suite without complications. At no time did robotic failure require the cancellation of a case or premature emergence from anesthesia.

\section{Conclusion}

Robotic prostatectomy is a safe and established procedure at our institution. Anesthestic management of these patients should take into consideration the medical co-morbidities associated with this patient population and medically optimize patients before surgery. Overall, patients tolerate the procedure well with minimal clinically significant cardiopulmonary effects. For patients with limited cardiopulmonary reserve, however, physicians must weigh these benefits with the negative cardiovascular and pulmonary changes associated with this type of procedure.

\section{References}

1. Jemal A, Siegel R, Ward E et al (2006) Cancer statistics. CA Cancer J Clin 56:84-103

2. Kantoff P, Taplin M (2005) Overview of the clinical presentation, diagnosis, and staging of prostate cancer. www.uptodate.com

3. Savarese D, Kupelian P, Klein E (2005) Overview of treatment of early prostate cancer. www.uptodate.com

4. Holmberg L, Bill-Axelson A, Helgesen F et al (2002) A randomized trial comparing radical prostatectomy with watchful waiting in early prostate cancer. N Engl J Med 347:781-789

5. Menon M, Shrivastava A, Tewari A (2005) Laparoscopic radical prostatectomy: conventional and robotic. Urology 66:101-104

6. Menon M, Tewari A, Peabody J et al (2004) Vattikuti institute prostatectomy, a technique of robotic radical prostatectomy for management of localized carcinoma of the prostate: experience of over 1100 cases. Urol Clin North Am 31:701-717

7. Ahlering T, Eichel L, Edwards R et al (2005) Impact of obesity on clinical outcomes in robotic prostatectomy. Urology 65:740744

8. Mokdad AH, Ford ES, Bowman BA et al (2003) Prevalence of obesity, diabetes and obesity-related health risk factors, 2001. JAMA 289:76-79

9. Shiga T, Wajima Z, Inoue $\mathrm{T}$ et al (2005) Predicting difficult intubation in apparently normal patients. Anesthesiology 103:429-437 
10. Sharma K, Brandstetter R, Brensilver J et al (1996) Cardiopulmonary physiology and pathophysiology as a consequence of laparoscopic surgery. Chest 110:810-815
11. Bhandari A, McIntire L, Kaul SA et al (2005) Perioperative complications of robotic radical prostatectomy after the learning curve. J Urol 174:915-918 